\title{
Editorial Note: Content-based Multimedia Indexing
}

Published online: 11 October 2018

(C) Springer Science+Business Media, LLC, part of Springer Nature 2018

Multimedia Tools and Applications gratefully acknowledges the editorial work of the scholars listed below on the special issue entitled, "Content-based Multimedia Indexing".

Of 34 papers submitted to this issue, 13 were eventually accepted after a stringent peerreview process.

Marco Bertin

Università di Firenze - MICC, Florence, Italy

marco.bertini@unifi.it

\section{Rita Cucchiara (Corresponding Guest Editor)}

Università di Modena e Reggio Emilia, Modena, Italy

rita.cucchiara@unimore.it

\section{Tao Mei}

Microsoft Research, Beijing, Beijing, China

tmei@microsoft.com

\author{
Alberto Del Bimbo \\ Università di Firenze - MICC, Florence, Italy \\ alberto.delbimbo@unifi.it
}

Publisher's Note Springer Nature remains neutral with regard to jurisdictional claims in published maps and institutional affiliations. 